\title{
UMA PROPOSTA PEDAGÓGICA PARA O USO DA MODELAGEM COMPUTACIONAL NO CURSO DE LICENCIATURA EM QUÍMICA DO CEFET CAMPOS.
}

Rodrigo Garrett da Costa, PGIE/UFRGS, CEFET Campos/RJ, garrett@ cefetcampos.br

Liliana Maria Passerino, PGIE/UFRGS, liliana@ pgie.ufrgs.br

\section{Resumo}

Relata-se a experiência no uso de um ambiente de simulação e modelagem computacional no ensino de Físico-Química. A pesquisa foi realizada com alunos do curso de Licenciatura em Química do Cefet Campos, RJ, e teve como objetivos: examinar a contribuição do uso do software Modellus no processo de ensino-aprendizagem; promover um ambiente colaborativo e cooperativo entre os estudantes; verificar as modificações na Zona de Desenvolvimento Proximal dos alunos proporcionadas por interações sociais nesse ambiente computacional. Os resultados obtidos nos testes indicaram uma melhora significativa na compreensão dos conceitos e das representações relativas aos modelos estudados, principalmente entre os alunos que obtiveram baixo rendimento no teste inicial.

Palavras-chave: Aprendizagem, Físico-Química, Lei dos Gases, Modelagem.

\section{A PEDAGOGICAL PROPOSAL FOR THE USE OF COMPUTATIONAL MODELLING IN THE CHEMISTRY EDUCATION COURSE AT CEFET- CAMPOS}

\begin{abstract}
This study presents an experiment on the use of a simulation and modelling computer environment in the teaching of Physical Chemistry. The research was conducted with students attending the Chemistry Teaching program at Cefet Campos aiming at: examining the contribution of using Modellus in the teaching and learning process, promoting a cooperative and collaborative learning environment among students, and observe changes in the student's Zone of Proximal Development promoted by social and cognitive interactions in that computational environment. The results obtained in the tests indicate a significant improvement in the understanding of the concepts and representations of the models investigated in the research, specially among low performance students who had bad results in the first test.
\end{abstract}

Keywords: Learning, Physical-Chemistry, Gas Laws, Modelling. 


\section{Introdução}

As dificuldades encontradas pelos estudantes durante a aprendizagem da FísicoQuímica têm sido atribuídas, em grande parte, à compreensão matemática dos fenômenos. Nesse sentido, Atkins (2003) sugere que o uso da matemática seja incentivado por sua aplicação na interpretação dos fenômenos físicos e químicos analisados.

Problemas relacionados ao ensino da Físico-Química também foram relatados por Lima (2004), Chou (2002), Silva et al. (2008), entre outros. Segundo esses autores, os alunos se preocupam mais em memorizar as equações do que em compreender os conceitos envolvidos. Para Santos (2002), estudar Físico-Química é algo que assusta os estudantes, seja pela complexidade exigida para a sua compreensão, pelo tratamento matemático envolvido, ou pela necessidade de associações do tipo imaginário-ideal-real sobre o assunto. De fato, como não conseguimos representar os fenômenos dinâmicos através de recursos estáticos como quadro e giz, torna-se necessário um grande poder de abstração por parte dos alunos.

Balen e Nets (2005) afirmam que as dificuldades dos estudantes podem ter como causa suas interpretações pessoais dos fenômenos que resultam de experiências no cotidiano, ou sua inabilidade de compreender o modelo e passar de um nível de representação a outro na interpretação do processo.

Contudo, toda a base da Química reside na Físico-Química, e sua importância têm crescido à medida que os métodos de análise computacional ficam mais sofisticados (Atkins, 2003). Nesse aspecto, muitos autores (Teodoro, 1997; Balen e Nets, 2005; Veit e Araújo, 2004) têm utilizado a simulação e a modelagem computacional na compreensão das leis e dos princípios físico-químicos, através da exploração visual dos modelos.

Um dos softwares educacionais de modelagem computacional que mais tem sido utilizado é o Modellus - um software livre, de fácil manuseio e boa interface gráfica. $\mathrm{O}$ programa foi desenvolvido pelo grupo de pesquisa do professor Vitor Duarte Teodoro (Teodoro, 1997), da Faculdade de Ciências e Tecnologia da Universidade Nova de Lisboa, para permitir a criação de diversos ambientes de aprendizagem, tanto para temas ligados às Ciências como à Matemática.

Veit e Araújo (2004) propuseram o emprego da modelagem computacional no ensino de Física utilizando o software Modellus na interpretação de gráficos da cinemática. Eles constataram que frequentemente os alunos utilizam expressões matemáticas sem que sejam feitos questionamentos sobre elas. Os autores consideram que, para o uso adequado dos modelos computacionais, é preciso que os alunos avaliem reflexivamente os conceitos físicos envolvidos e também as relações existentes entre eles.

Outro autor que sugeriu o uso do software Modellus em atividades conjuntas de exploração e criação de animações interativas no ensino de Física foi Rodrigues (2005), em sua pesquisa de Mestrado em Educação. Seu trabalho foi desenvolvido com estudantes do curso de Redes de Acessos em Telecomunicações do $3^{\circ}$ grau do CEFET-PB, no eixo temático oscilações, com o objetivo de avaliar a dinâmica de aquisição do conhecimento dos aprendizes. 
O presente artigo apresenta uma discussão teórico-prática sobre o uso do software Modellus na disciplina de Físico-Química do curso de Licenciatura em Química do Cefet Campos com os seguintes objetivos: (1) examinar a contribuição da simulação e modelagem computacional no aprendizado de Físico-Química, no eixo temático estudo dos gases; (2) promover um ambiente colaborativo e cooperativo entre os estudantes na realização da atividade computacional; (3) verificar se a interação social no ambiente computacional potencializa a atuação na Zona de Desenvolvimento Proximal (ZDP) dos sujeitos envolvidos. Para tanto, remetemo-nos aos pensamentos de Vygotsky (1999), ao considerar a aprendizagem como um processo social de mudanças na natureza humana, no qual os sujeitos constroem seus conhecimentos através das interações com o meio.

$\mathrm{Na}$ seção 2, são abordados os conceitos de modelo e modelagem computacional, referindo-se ao uso do software Modellus como instrumento de mediação, sob a concepção sócio-histórica de Vygotsky voltada à construção do conhecimento. $\mathrm{Na}$ seção 3, são descritos os materiais e métodos utilizados na pesquisa. Na seção 4, são apresentados os dados obtidos, bem como as discussões dos resultados alcançados. Finalmente, na seção 5, são destacadas as conclusões da pesquisa.

\section{Referencial Teórico}

\subsection{Modelos e Modelagem Computacional}

A Física e a Química, enquanto ciências, buscam explicação para os fenômenos naturais através do método científico. Por meio de experiências, observações e medidas, são construídas hipóteses que, uma vez testadas e comprovadas, geram os modelos. Os modelos são formas essencialmente matemáticas de representação dos fenômenos que nos fornecem uma aproximação da realidade.

Greca e Moreira (2002), citados por Veit e Araújo (2004) definem o modelo científico como sendo uma descrição simplificada de um sistema físico idealizado, aceito pela comunidade científica. No caso da Física, essa descrição envolve tanto proposições semânticas quanto modelos matemáticos subjacentes. Tais modelos científicos servem como ponte entre o mundo real (complexo e holístico) e um mundo idealizado, existente apenas na mente dos cientistas. Esse modelo simplificado preserva as características essenciais do sistema ou fenômeno que se pretende descrever, explicar ou predizer.

O gás perfeito é um modelo idealizado do estado gasoso da matéria e o ponto de partida para a discussão dos gases reais. Embora seja útil em algumas condições, o modelo ideal falha em muitas situações estudadas. Nesses casos, outros modelos devem ser utilizados para representar adequadamente o comportamento dos sistemas gasosos por meio de cálculos que mais os aproximem da realidade. Assim sendo, a modelagem computacional pode ser uma ferramenta extremamente valiosa para a interpretação das diversas equações que descrevem o comportamento dos gases reais, como: van der Waals, Berthelot, e Dieterici ${ }^{1}$.

\footnotetext{
1 Essas equações foram propostas por seus criadores para corrigir os desvios da idealidade através da utilização de parâmetros empíricos.
} 
Para Teodoro (1997), a criação e exploração de um modelo é uma experiência importante no processo de aprendizagem, pois descreve a maneira como funciona a natureza. Segundo esses autores, as práticas educativas de modelagem computacional podem apresentar características de poderosos ambientes de aprendizagem, onde:

- O conhecimento encontra-se contextualizado;

- As interações entre os indivíduos são estimuladas, propiciando discussões sobre os temas propostos;

- As diferentes formas de representação (equações e gráficos) facilitam a construção das relações entre as variáveis;

- Ocorrem motivações intrínsecas nos processos de aprendizagem;

- Os objetos formais podem ser manipulados como entidades reais.

Dessa forma, o uso da simulação e da modelagem computacional no ensino representa uma forma de repensar e interagir com os modelos científicos, na busca de melhores caminhos para a compreensão de muitos fenômenos físico-químicos.

\subsection{O Modellus como instrumento de mediação no ensino de Físico-Química}

Conforme mencionado na seção 1, o Modellus é um software educacional que foi originalmente criado para a utilização em modelagens computacionais, embora também possa ser utilizado para criar simulações. Se o analisarmos a partir da teoria sócio-histórica de Vygotsky (1999), esse software pode ser considerado tanto um instrumento quanto um signo, devido à sua função mediadora no processo de ensino-aprendizagem. Através dele, é possível manipularmos objetos do domínio da natureza com o intuito de mudá-los. Mas segundo esse autor, o controle da natureza e o controle do comportamento estão mutuamente ligados, de forma que e a alteração provocada pelo homem na natureza altera também a própria natureza do homem.

A forma de orientação do comportamento humano é a principal diferença entre instrumento e signo. Enquanto o primeiro provoca mudanças sobre os objetos, ou seja, age externamente, o segundo constitui um meio de atividade interna dirigido para o controle do próprio indivíduo. À medida que o aprendiz interage com o software de simulação, programando-o com o objetivo de conduzir um processo físico ou químico, ele está simplesmente tentando dominar os processos naturais. Mas a partir do momento que esses objetos começam a provocar alterações no indivíduo, alterando seu comportamento, dizemos que está ocorrendo a internalização, que segundo Vygotsky (1999), é uma operação que representa a transformação de um processo interpessoal em intrapessoal. Primeiramente ela ocorre no nível social (entre pessoas), para depois passar ao nível individual (interior do indivíduo).

A interação social representa um elemento necessário ao processo de aprendizagem e desenvolvimento do indivíduo. Isso implica na participação ativa dos sujeitos no processo de troca, caracterizado por diferentes níveis de experiências e conhecimentos. Logo, quando ocorre uma atividade em grupo, dois níveis de desenvolvimento poderão ser 
identificados: o real e o potencial. A ZDP é explicada por Vygotsky (1999, p. 112) como sendo:

[...] a distância entre o nível de desenvolvimento real que se costuma determinar através da solução independente de problemas, e o nível de desenvolvimento potencial, determinado através da solução de problemas sob a orientação de um adulto ou em colaboração com companheiros mais capazes.

Nesse sentido, tornam-se necessários estudos que proponham metodologias de ensino apoiadas no uso de recursos computacionais com vistas à estimulação da ZDP dos alunos.

\section{Materiais e Métodos}

O estudo foi realizado no primeiro semestre de 2008 com todos os 13 alunos matriculados no sexto período do curso de Licenciatura em Química do Cefet Campos. Em um primeiro momento, buscou-se avaliar os conhecimentos prévios desses estudantes através da aplicação de um pré-teste constando de cinco questões conceituais, realizadas individualmente e sem consulta ao conteúdo previamente abordado. As questões apresentaram o mesmo valor e versaram sobre a representação e interpretação das equações de estado dos gases, bem como os conceitos de fator de compressibilidade e temperatura crítica. Adicionalmente, solicitou-se que os alunos julgassem suas respostas como corretas, meio certas ou erradas. Buscou-se, com isso, obter informações sobre as habilidades metacognitivas dos estudantes, através do cálculo dos índices KMA e KMB, propostos por Pimentel et al (2005).

Os índices KMA (Knowledge Monitoring Accuracy - Precisão do Monitoramento do Conhecimento) e o KMB (Knowledge Monitoring Bias - Desvio do Monitoramento do Conhecimento) foram criados por Tobias e Everson (2002) e sugeridos por Pimentel et al. (2005) como uma proposta de avaliação adaptativa da aprendizagem, baseada no perfil cognitivo e metacognitivo do aluno. O primeiro mede a diferença entre o conhecimento que o aprendiz julga possuir, num certo domínio particular, e seu conhecimento real constatado em seu desempenho nas avaliações. Já o segundo é utilizado para identificar o tipo de desvio do aprendiz no julgamento de seu conhecimento, ou seja, se o aluno comporta-se de forma otimista, pessimista, realista ou aleatória. O estabelecimento da relação entre esses dois índices nos permite saber se o aluno tem consciência do próprio nível de conhecimento, ou seja, se ele sabe determinar o seu conhecimento metacognitivo.

Segundo Tobias e Everson (2002), o monitoramento dos conhecimentos prévios é um processo metacognitivo fundamental para que os estudantes consigam se comprometer em atividades avançadas, como avaliar, planejar e controlar a própria aprendizagem. Dessa forma, este estudo buscou traçar uma relação entre a evolução do perfil metacognitivo dos estudantes na realização das atividades de simulação computacional, e seu desempenho nos testes, a partir das informações obtidas com o cálculo dos índices KMA e KMB.

Posteriormente à realização do pré-teste pelos alunos, foi feito um breve intervalo para a correção das questões. À medida que regressavam, os estudantes eram organizados 
em duplas em função de suas notas na avaliação, de modo que um aluno com alto rendimento no teste ficava sempre com um aluno de baixo rendimento. Com isso, pretendeu-se promover o desenvolvimento das funções ainda não amadurecidas nos indivíduos, através das interações sociais e cognitivas.

A partir de então, foi realizada uma aula expositiva de aproximadamente 30 minutos sobre o software, suas ferramentas e funcionalidades. Enquanto assistiam à apresentação, os alunos acompanhavam e repetiam os mesmos passos dados pelo professor. Quando se percebeu que os estudantes dominavam razoavelmente o software, foi entregue um roteiro de atividades propondo a construção de modelos computacionais baseados nas equações de estado dos gases e suas respectivas representações gráficas. Nos 60 minutos subsequentes, preocupamo-nos em observar as interações entre as duplas no cumprimento das tarefas.

Inicialmente, procurou-se orientar os alunos na representação dos modelos, valorizando o uso das ferramentas matemáticas e gráficas disponíveis no software. Nesse momento, todas as duplas seguiram o proposto no roteiro, conforme pode ser observado na Figura 1. Mais adiante, buscou-se valorizar a capacidade de criação e análise dos alunos através da construção de modelos e o questionamento da sua validade (Figura 2).

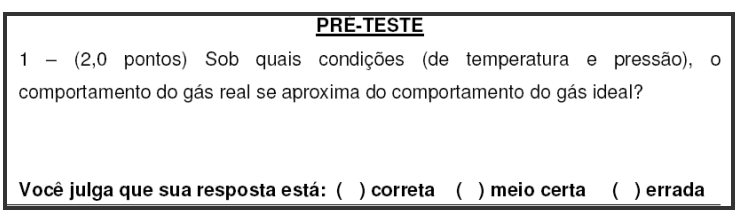

Figura 1 - Proposta de representação do modelo no roteiro de atividades.
8 - Sugira uma terceira equação que leve em consideração os parâmetros empíricos "a" e "b" e faça o seu gráfico. Então explique se esta equação pode representar o comportamento de um gás real.

Figura 2 - Proposta de criação do modelo no roteiro de atividades.

Ao término da atividade de simulação computacional, foi aplicado um pós-teste, reproduzindo as mesmas questões do pré-teste e adotando-se os mesmos critérios, com o objetivo de verificar se houve avanço no entendimento dos conceitos estudados. Por último, sugeriu-se que os alunos preenchessem um questionário de avaliação das atividades, com o objetivo de capturar suas impressões sobre a aula e o uso do software. O questionário tratou de questões sobre o uso do computador, do trabalho em duplas, do papel do professor como mediador das atividades, da facilidade no uso do software e de sua importância no ensino de Físico-Química.

A consolidação dos dados obtidos no estudo apoiou-se tanto em aspectos qualitativos quanto quantitativos, por entendermos que as pesquisas realizadas no campo da pedagogia são perfeitamente compatíveis com o tratamento matemático das informações. Castro (2006) é de opinião que:

[...] há uma fertilização cruzada virtuosa entre os métodos qualitativos e quantitativos. Cada um chega onde o outro não consegue chegar. $\mathrm{O}$ método quantitativo pergunta "como" acontece, "o que" acontece. Já o método qualitativo pergunta "porque" acontece.

No que diz respeito ao aspecto qualitativo de nossa pesquisa, ele se baseou na observação participativa, onde o professor é ao mesmo tempo observador e ator, fazendo o 
experimento funcionar e explicando os fenômenos envolvidos. Quanto ao aspecto quantitativo da pesquisa, foram analisados os dados individuais e as medidas de tendência central, assim como de dispersão, para medir a evolução no rendimento dos alunos por meio de suas notas nos testes. Além disso, pretendeu-se estabelecer uma relação entre a evolução do desempenho dos alunos na resolução dos problemas e seus perfis metacognitivos, através da aplicação do teste de correlação de Pearson $(r)$.

\section{Resultados e Discussão}

Com base na avaliação feita através do pré-teste, cerca de $70 \%$ dos alunos ficaram com um desempenho nas respostas menor do que 6,0, num total de 10,0 pontos. Após a aula de simulação computacional com o software Modellus, o percentual de notas registradas abaixo dos 6,0 pontos caiu para apenas 30\%. Além disso, verificou-se que, com base nas avaliações, aproximadamente $85 \%$ dos alunos tiveram seu desempenho melhorado após essa aula (Figura 3). Resultados semelhantes foram encontrados por Balen e Nets (2005) na pesquisa realizada com alunos dos cursos de Licenciatura em Química e Engenharias, utilizando a modelagem computacional aplicada ao estudo dos gases.

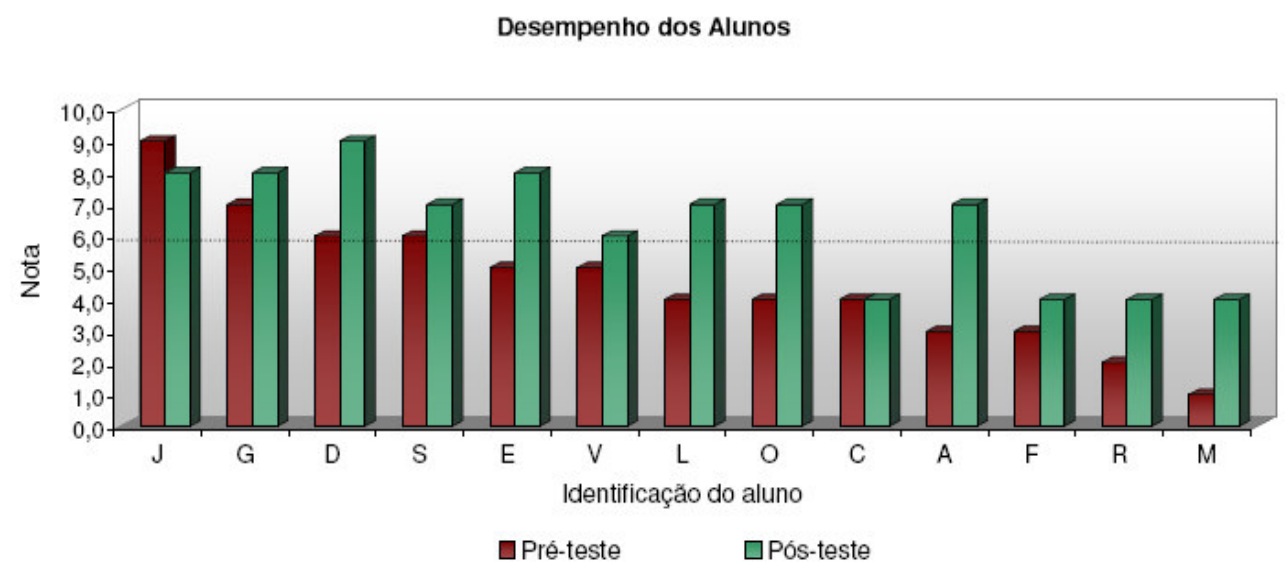

Figura 3 - Evolução nos rendimentos dos alunos nos testes.

Ainda em relação ao desempenho dos estudantes nos testes, pode-se verificar na Figura 4 que houve um aumento significativo no valor da sua média, que passou de 4,5 para 6,4. Quando analisamos os valores da nota mais baixa do pré-teste, comparativamente ao pós-teste, também percebemos uma melhora expressiva, uma vez que passou de 1,0 no préteste para 4,0 no pós-teste. Além disso, houve uma pequena melhora no desvio padrão das notas em relação a media, que passou de 2,1 para 1,8, o que indica ter havido uma aprendizagem mais homogênea. Quando a evolução no desempenho dos alunos é analisada levando-se em consideração as interações existentes nas duplas, nota-se um progresso mais significativo ocorrendo entre aqueles que tiraram as menores notas no pré-teste, ou seja, aqueles representados pelas letras O, M, A, E, F e R (Figura 5). 


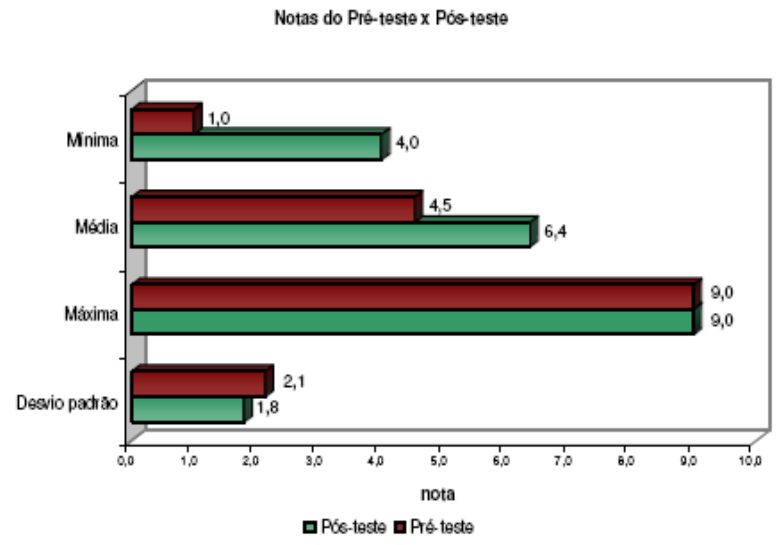

Figura 4 - Medidas de tendência aplicadas ao rendimento dos alunos nos teste.

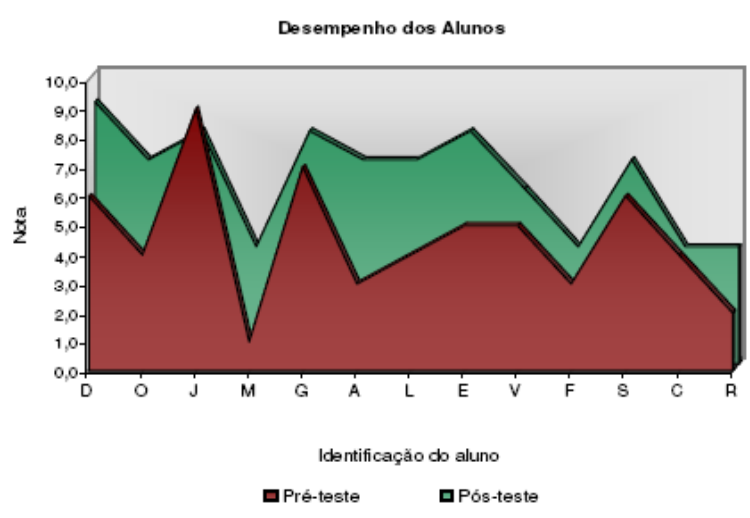

Figura 5 - Evolução dos rendimentos das duplas de alunos nos testes.

A respeito da análise do perfil metacognitivo, os dados apresentados na Figura 6 indicam a predominância do KMA médio (entre $-0,25 \mathrm{e}+050$ ). Isso quer dizer que algumas vezes os alunos estimaram corretamente seus conhecimentos, mas frequentemente cometeram erros no julgamento. Essa figura ainda permite fazer certas leituras individuais a respeito de alguns estudantes: o aluno "A" estimou corretamente seu conhecimento na maioria das vezes, obtendo assim um KMA alto (entre + 0,50 e $+1,0$ ); o aluno "L" acertou todas as previsões dos pós-teste a respeito do erro ou acerto das questões (KMA $=+1,0)$; já o aluno "O" obteve um KMA médio no pré-teste, mas no pós-teste, seu KMA foi classificado como alto. Com relação ao índice $\mathrm{KMB}$, ficou constatado que a grande maioria dos alunos agiu aleatoriamente (KMB entre - 0,25 e + 0,25), ou seja, ora estimavam seus conhecimentos de maneira otimista, ora de maneira pessimista.

\begin{tabular}{|c|c|c|c|c|c|c|c|c|c|}
\hline \multirow[b]{2}{*}{ IA } & \multicolumn{2}{|c|}{ nota } & \multirow[b]{2}{*}{ variação } & \multicolumn{2}{|c|}{ KMA } & \multirow[b]{2}{*}{ variação } & \multicolumn{2}{|c|}{ KMB } & \multirow[b]{2}{*}{ variação } \\
\hline & Pré-teste & Pós-teste & & Pré-teste & Pós-teste & & Pré-teste & Pós-teste & \\
\hline $\mathrm{J}$ & 9,0 & 8,0 & $-1,0$ & 0,40 & 0,10 & $-0,3$ & $-0,20$ & $-0,10$ & 0,1 \\
\hline D & 6,0 & 9,0 & 3,0 & 0,40 & 0,40 & 0,0 & 0,20 & 0,00 & $-0,2$ \\
\hline G & 7,0 & 8,0 & 1,0 & 0,40 & 0,40 & 0,0 & $-0,20$ & $-0,20$ & 0,0 \\
\hline$E$ & 5,0 & 8,0 & 3,0 & 0,40 & 0,40 & 0,0 & $0, \infty$ & 0,00 & 0,0 \\
\hline $\mathrm{s}$ & 6,0 & 7,0 & 1,0 & 0,40 & $-0,20$ & $-0,6$ & $0, \infty$ & 0,20 & 0,2 \\
\hline $\mathrm{L}$ & 4,0 & 7,0 & 3,0 & 0,70 & 1,00 & 0,3 & $-0,10$ & 0,00 & 0,1 \\
\hline 0 & 4,0 & 7,0 & 3,0 & 0,00 & 0,70 & 0,7 & 0,20 & 0,10 & $-0,1$ \\
\hline A & 3,0 & 7,0 & 4,0 & 0,70 & 0,70 & 0,0 & $-0,10$ & $-0,10$ & 0,0 \\
\hline $\mathrm{V}$ & 5,0 & 6,0 & 1,0 & $-0,20$ & 0,10 & 0,3 & 0,40 & 0,30 & $-0,1$ \\
\hline $\mathrm{C}$ & 4,0 & 4,0 & 0,0 & 0,10 & 0,40 & 0,3 & 0,10 & 0,20 & 0,1 \\
\hline $\mathrm{F}$ & 3,0 & 4,0 & 1,0 & 0,40 & 0,40 & 0,0 & 0,00 & 0,00 & 0,0 \\
\hline $\mathrm{R}$ & 2,0 & 4,0 & 2,0 & $-0,20$ & 0,10 & 0,3 & 0,20 & $-0,10$ & $-0,3$ \\
\hline M & 1,0 & 4,0 & 3,0 & 0,40 & 0,40 & 0,0 & 0,00 & 0,00 & 0,0 \\
\hline
\end{tabular}

Figura 6 - Comparação entre as notas dos testes, os índices KMA e KMB.

Através dos dados apresentados na Figura 6, foi possível calcular os coeficientes de correlação de Pearson quando confrontamos a evolução das notas do pré-teste e do pósteste com as variações do KMA e do KMB calculadas, também, nesses dois momentos. O 
coeficiente $r$ calculado a partir das variações entre as notas dos testes e as variações de KMA ficou em $+0,17$, ou seja, existe uma fraca relação direta entre eles. Já o coeficiente $r$ calculado a partir das variações entre as notas dos testes e as variações de KMB apresentou um valor de - 0,35, revelando a existência de uma correlação moderadamente inversa.

Quanto ao preenchimento do questionário de avaliação pelos alunos a respeito das atividades, verificou-se que: $85 \%$ dos alunos nunca tinham experimentado uma aula de Físico-Química com o recurso computacional; o mesmo percentual preferiu trabalhar esse tipo de aula em duplas e com a presença do professor do que individualmente; os estudantes foram unânimes em considerar necessário o uso da simulação computacional no ensino de Físico-Química e também em afirmar que o software é de fácil manipulação.

\section{Conclusões}

Os resultados obtidos nos testes, juntamente com as observações realizadas, apresentam evidências de que a incorporação do Modellus ao estudo da Físico-Química melhorou a compreensão dos conceitos e das representações matemáticas dos modelos de gases ideais e reais. Através da utilização do software, foi possível promover uma aprendizagem colaborativa e reflexiva, a qual repercutiu de forma positiva, principalmente no rendimento dos alunos que obtiveram baixo resultado no pré-teste. Tal fato deve estar associado à participação ativa dos estudantes no processo de troca de experiência e conhecimentos. Dessa forma, podemos afirmar que a cooperação e a colaboração funcionaram como mecanismos sócio-cognitivos favoráveis ao processo de ensinoaprendizagem, uma vez que provocaram modificações na ZDP desses alunos. Cabe destacar aqui a importância na atuação do professor no planejamento e na execução da proposta pedagógica, além de sua participação como observador e mediador do processo de construção do conhecimento.

A respeito da análise dos índices KMA e KMB, os dados coletados na pesquisa não foram suficientes para evidenciar uma relação significativamente positiva entre a melhora na aprendizagem dos estudantes e o aumento das suas habilidades metacognitivas. Talvez porque essas mudanças cognitivas não ocorram tão rapidamente a ponto de serem detectadas imediatamente, ou porque o tamanho da população analisada não tenha sido estatisticamente suficiente.

\section{Referências Bibliográficas}

1. ATKINS, Peter W; PAULA, Julio de. Físico-Química. $7^{\circ}$ ed, Rio de Janeiro: Livros Técnicos e Científicos, 2003. 356 p.

2. BALEN, O.; NETZ, P. A. Utilizando a modelagem e a simulação computacional no estudo dos gases ideais e reais. In: XVI Simpósio Nacional de Ensino de Física, 2005, Rio de Janeiro. Anais. Rio de Janeiro: Sociedade Brasileira de Física (SBF) / CEFET- RJ. 2005.

3. CASTRO, Cláudio de Moura. A prática da pesquisa. $2^{\circ}$ ed., São Paulo: Pearson Prentice Hall, 2006. 190 pág. 
4. CHOU, C. Y. Science Teachers' Understanding of Concepts in Chemistry. Proc. Natl. Sci. Counc. ROC(D), v. 12, n 2 2, 2002. p. 73-78

5. LIMA, D. M. L. Dificuldades de aprendizagem na área de Física: análise da realidade na E.E.M. Liceu de Tauá - Lili Feitosa. Centro de Educação, Ciência e Tecnologia da Região dos Inhamuns: Tauá, Universidade Estadual do Ceará. 2004. 72p. Monografia em Educação Especial.

6. PIMENTEL, E. P.; OMAR, N.; FRANÇA, V. F. Um Modelo para Incorporação de Automonitoramento da Aprendizagem em STI. Revista Brasileira de Informática na Educação, v. 13, n. 1, mar. 2005.

7. RODRIGUES, G. L. Animação interativa e construção dos conceitos da física: trilhando novas veredas pedagógicas. Universidade Federal da Paraíba, João Pessoa, 2005. 108p. Dissertação em Educação.

8. SANTOS, S. C. Modelagem de cenários telemáticos como estratégia cognitiva para trabalhar conceitos físico-químicos: indicadores de aprendizagem. Universidade Federal do Rio Grande do Sul, Porto Alegre, 2002. 389p. Tese de Doutorado em Informática na Educação.

9. SILVA, J. R. M.; MOURA, S. A. S.; SOAR, R. S. Levantamento das dificuldades dos professores no ensino de Química em escolas de nível Médio de Campo Grande - MS. In: XIV Encontro Nacional de Ensino de Química (XIV ENEQ), 2008, Curitiba. Anais. Sociedade Brasileira de Química (SBQ) / UFPA. 2008.

10. TEODORO, V.D. Modelação computacional em Ciências e Matemática. Revista Brasileira de Informática na Educação. v.10 , n. 2, 1997. Uniandes-Lidie, Colombia. p. 171182. Disponível em: <http://www.colombiaaprende.edu.co/html/mediateca/1607/articles112586_archivo.pdf>. Acesso em 10 mai. 2008.

11. TOBIAS, S.; EVERSON, H. T. Knowing What You Know and What You Don't: Further Research on Metacognitive Knowledge Monitoring. College Board Research Report $N^{\circ}$. 2002-3, College Entrance Examination Board, New York, 2002. Disponível em: $<$ http://www.collegeboard.com/research/pdf/071623RDCBRpt02-3.pdf>. Acesso em: 13 mar. 2007.

12. VYGOTSKY, Lev S. A formação social da mente. São Paulo: Martins Fontes, 1999.191p.

13. VEIT, E. A; ARAÚJO, I. S. Modelagem no Ensino de Física. Revista Educação, Maceió, Alagoas, v. 13, n. 21, p. 51-70, 2004. 